\title{
Total phenolic content and antioxidant activity of sesame cake aqueous extracts
}

\author{
Yen T. X. Nguyen ${ }^{1}$, Costas Stathopoulos ${ }^{2}$, Chockchaisawasdee Suwimol ${ }^{2}$, \\ Phuong L. Nguyen ${ }^{3}$, \& Tuyen C. Kha ${ }^{1 *}$ \\ ${ }^{1}$ Faculty of Food Science and Technology, Nong Lam University, Ho Chi Minh City, Vietnam \\ ${ }^{2}$ Faculty of Bioscience Engineering, Ghent University Global Campus, South Korea \\ ${ }^{3}$ American International School, Ho Chi Minh City, Vietnam
}

ARTICLE INFO
Research Paper
Received: November 05, 2018
Revised: December 18, 2018
Accepted: December 26, 2018
Keywords
Antioxidant activity
Microwave pre-treatment
Sesame cake
Total phenolic content
*Corresponding author
Kha Chan Tuyen
Email: khachantuyen@hcmuaf.edu.vn

Cited as: Nguyen, Y. T. X., Stathopoulos, C., Suwimol, C., Nguyen, P. L., \& Kha, T. C. (2018). Total phenolic content and antioxidant activity of sesame cake aqueous extracts. The Journal of Agriculture and Development 17(6), 77-84.

\begin{abstract}
The objective of this study was to investigate the efficiency of phenolic extraction and antioxidant activity from sesame cake using water extraction method and to evaluate the possibility of employing microwave irradiation to improve the extraction yield. The result showed that extraction temperature had major influence on total phenolic content and antioxidant activities of the extracts, whereas extraction time was found to be insignificant. The optimum extraction condition recommended were $90^{\circ} \mathrm{C}$ for $30 \mathrm{~min}$ in this research. Furthermore, microwave pre-treatment at $120 \mathrm{~s}$ could have significantly positive influence on the overall extraction yield, especially the total phenolics and antioxidants based on FRAP assay. Therefore, the obtained results suggest that sesame aqueous extracts could be a source of antioxidants with more feasible applications in food as well as other industries.
\end{abstract}

\section{Introduction}

The potential applications of antioxidants, the consumer preferences of totally "natural" products, and the drawbacks of synthetic antioxidants, all has risen an emerging trend to look for a new source of antioxidants which can satisfy both the safety concerns and economic hindrance. Accordingly, the extraction of antioxidants from natural resources, especially from the utilization of residual products or waste, is recently of high considerations by many researchers and manufacturers. In several last decades, numerous studies and researches on such kinds of materials for their antioxidant composition, properties, and capacity, such as the studies of Guo et al. (2003), Pan et al. (2003), Wang et al. (2015) on the pulp, peel and seed parts of various kinds of fruits, those of Pinelo et al. (2005), Candrawinata et al. (2014), Saikia et al. (2015) on various sources of fruit pomace, and of Wanasundara et al. (1995), Suja et al. (2005), Terpinc et al. (2012) on different oilseed cake extracts, have been conducted with promising results.

Following up the current interests, sesame oil cake, which has been normally referred to as a byproduct after sesame oil pressing and extraction process and is currently used as cattle feed, has been increasingly investigated and utilized as a potent source of bioactive compounds. The enormous amount of sesame cake produced annually by the oil-pressing process makes it interesting to evaluate what remains in the cake after the extraction process as well as to utilize the cake 
as a source of value-added materials instead of a source of poultry feed. The antioxidant extraction and characteristics of sesame meal has already conducted by interested scientists and researchers. Yet, most of previous researches have not focused on the potential of aqueous phenolic, but the organic solvent extraction which employed organic solvents, hexane, methanol and ethanol were most frequently used, instead. In some cases, a mixture of solvents could be employed to optimize the extraction yield (Mohdaly et al., 2010; Reshma et al., 2013). The main drawbacks of organic solvents, however, are their high flammability and their potential hazards for both human health and environment. Moreover, solvent extraction also requires further steps for evaporation or removal of solvent to recover the extracts (Joana Gil-Chávez et al., 2013). Therefore, a search for an alternative which could minimize those merits of organic solvents has been continued for years.

Generally recognized as a green, safe, and cheap extraction solvent, water is recently referred to as a brilliant alternative for other organic solvents, which are usually toxic, volatile, and highly flammable. Another advantage of water-based extraction is that aqueous extracts do not require further concentration or separation steps as to the solvent extracts, which is sometimes very expensive and time-wasting process. The tendency of selecting water as the only solvent for extraction is evidently potential through the results of many recent (Çam \& Aaby, 2010; Candrawinata et al., 2014). Moreover, in case of employing microwave as a pre-treatment method, the presence of water could facilitate the effectiveness of microwave irradiation in terms of phenolic recovery efficiency (Veggi et al., 2013). Microwave irradiation were selected as the pre-treatment method and compared with the effect of ultrasonic pre-treatment on sesame cake extraction in other unpublished papers. Microwave has been proposed by many researchers as a brilliant pretreatment candidate because of its effectiveness, simplicity and greatly lower time processing requirement (Azmir et al., 2013).

As a result, the objective of this present study was to investigate the efficiency of phenolic extraction as well as the antioxidant capacity from sesame cake through water extraction method. Furthermore, the work also focused on the effect and interactions of extraction temperature and time by the full factorial experiment design as well as the possibility of employing microwave irradiation as a pre-treatment method to improve the extraction yield.

\section{Materials and Methods}

\subsection{Chemicals}

All redox reagents, gallic acid and Trolox (6-hydroxy-2,5,7,8-tetramethylchroman-2carboxylic acid) were of analytical grade and were purchased from Sigma Aldrich Laboratory Chemicals.

\subsection{Aqueous extraction of phenolic con- stituents from sesame cake}

Sesame cake $(0.40 \pm 0.02 \%)$, after getting from commercial sesame oil producer, was stored at $18^{\circ} \mathrm{C}$ until sampling. The cake was extracted in which water was the only solvent by preparing sample at the ration of $0.05 \mathrm{~g}$ sample/ $\mathrm{mL}$ distilled water, and then, placing and shaking gently in a water bath (model: JSSB-30T, South Korea) which was pre-set at an assigned temperature $\left(40,50,60,75,90^{\circ} \mathrm{C}\right)$ and time $(30,45,60$ min). The extracted mixture was cooled in water bath for $10 \mathrm{~min}$ so as to stabilize the phenolic extracted. The mixture was continuously centrifuged at $3500 \mathrm{rpm}$ for $20 \mathrm{~min}$ and was filtered through Whatman filter paper to obtain the filtrate considered as the phenolic extract of sesame cake.

\subsection{Pre-treatment with microwave on aque- ous extraction of sesame cake}

The previous experiment was determined the optimum condition for aqueous extraction of sesame cake. The further step was to investigate the effect of microwaves as pre-treatment method on the total phenolic yield and antioxidant capacity of aqueous sesame cake extract. The microwave model employed was LG MW233SK. The microwave power and frequency were set at $700 \mathrm{~W}$ and $2450 \mathrm{MHz}$ respectively while pretreatment time was the variable parameter with 5 levels of interest $(0,30,60,90,120 \mathrm{~s})$. The levels were referenced from previous work that successfully applied microwave as pre-treatment method for extraction. Samples, after being pre-treated, continuously experimented under the optimum 
extraction condition selected by the previous part of the research.

\subsection{Analytical methods}

\subsubsection{Determination of moisture content}

The moisture content of the sesam cake was determined by drying at a temperature of $105^{\circ} \mathrm{C}$ until a constant weight was reached.

\subsubsection{Determinations of the total phenolic content and the antioxidant activity}

- Total phenolic content (TPC): TPC was determined by employing the Folin-Ciocalteu's colorimetric assay which was adapted from Thaipong et al. (2006) with some modifications. $150 \mu \mathrm{L}$ of sesame cake extract was reacted with $150 \mu \mathrm{L}$ of Folin Ciocalteu reagent (0.25) for 2 min, then $2400 \mu \mathrm{L}$ of sodium carbonate $(5 \% \mathrm{w} / \mathrm{v})$ was added and mixed evenly using a Vortex. Absorbance was measured at $765 \mathrm{~nm}$ using spectrophotometer after the mixture was incubated for $1 \mathrm{~h}$ after mixing. The results were expressed in gallic acid equivalents (GAE, mg/g dry sample).

- Antioxidant activity measurement: The three assays, ABTS, DPPH, and FRAP, were optioned to measure antioxidant activity of the extracts.

$\diamond$ ABTS radical cation decoloration assay: The ABTS assay was employed with minor modifications from the method of Arnao et al. (2001) and Thaipong et al. (2006). The stock solution was ABTS $^{+}$solution $(7.4 \mathrm{mM})$ and potassium persulfate solution $(2.6 \mathrm{mM})$. The working solution was prepared fresh every assay by mixing the two stock solutions at the volume ratio of $1: 1$ and was incubated in the dark at ambient temperature for 12-15 h before methanol was then added for dilution purpose at the ratio of $1: 45(\mathrm{v} / \mathrm{v})$ of the working solution and methanol respectively so as to the mixture get an absorbance of 1.1 \pm 0.02 units when measured at $734 \mathrm{~nm}$ by using the spectrophotometer. $150 \mu \mathrm{L}$ of the sesame cake extract reacted with $2850 \mu \mathrm{L}$ of ABTS solution added. The mixture was mixed well by using a Vortex and incubated for $2 \mathrm{~h}$ in dark room. Standard curve was prepared for the concentration of Trolox ranging from 100 to $600 \mu \mathrm{M}(0.025-0.9$ $\mathrm{mg} \mathrm{TE} / \mathrm{ml}$ water). The result was expressed as $\mathrm{mg}$ of Trolox equivalent (TE)/ml of water, which was then transferred to $\mathrm{mg} \mathrm{TE} / \mathrm{g}$ of dry sample. $\diamond$ DPPH radical scavenging activity assay: The method to evaluate antioxidants using DPPH assay following the adapted method of Candrawinata et al. (2014), which was modified from method of Brand-Williams et al. (1995) with some modifications. Stock solution was prepared by dissolving $24 \mathrm{~g}$ DPPH reagent in $100 \mathrm{ml}$ methanol. The solution was refrigerated at $-20^{\circ} \mathrm{C}$ before and after used. The working solution was prepared fresh every day of experiment by mixing stock solution and methanol at the ration 10:45 $(\mathrm{v} / \mathrm{v})$, respectively until the solution gained the absorbance of $1.1 \pm 0.02$ units. The absorbance was measured at $515 \mathrm{nM}$ by spectrophotometer. $150 \mu \mathrm{L}$ of the sesame cake extract reacted with $2850 \mu \mathrm{L}$ of DPPH working solution added. The mixture was mixed well by using a Vortex and incubated for $30 \mathrm{~min}$ in dark room. Standard curve was prepared for the concentration of Trolox ranging from 100 to $800 \mu \mathrm{M}(0.025-2.0$ $\mathrm{mg} \mathrm{TE} / \mathrm{ml}$ water). The result was expressed as $\mathrm{mg}$ of Trolox equivalent (TE) $/ \mathrm{ml}$ of water, which was then transferred to mg TE/g of dry sample.

$\diamond$ Ferric reducing/antioxidant power (FRAP) assay: The FRAP assay was adapted the method of Candrawinata et al. (2014) which modified from that of Thaipong et al. (2006) and Benzie and Strain (1996). Stock solutions was obtained by preparing three solution: acetate buffer (300 $\mathrm{mM}-\mathrm{pH}=3.6)$, TPTZ $(10 \mathrm{mM})$ in $\mathrm{HCl}(40 \mathrm{mM})$ solution and $\mathrm{FeCl}_{3} \cdot \mathrm{H}_{2} \mathrm{O}(20 \mathrm{mM})$ solution. Water bath prepared at $50^{\circ} \mathrm{C}$ was employed as preparing TPTZ $10 \mathrm{mM}$ in $\mathrm{HCl} 40 \mathrm{mM}$ solution to dissolve them well. The working solution was prepared fresh when used, at the ratio of $10 \mathrm{ml}$ acetate buffer, $1 \mathrm{~mL}$ TPTZ and $1 \mathrm{~mL} \mathrm{FeCl} 3 \cdot \mathrm{H}_{2} \mathrm{O}$. Incubation time was set for $1 \mathrm{~h}$ before being available for reaction. After incubation, $150 \mu \mathrm{L}$ of the sesame cake extract was added $2850 \mu \mathrm{L}$ of repared FRAP working solution, mixed well and incubated for 30 min in dark room. Standard curve was prepared for the concentration of Trolox ranging from 100 to $800 \mu \mathrm{M}(0.025-2.0 \mathrm{mg} \mathrm{TE} / \mathrm{ml}$ water $)$. The result was expressed as mg of Trolox equivalent (TE) $/ \mathrm{ml}$ of water, which was then transferred to mg TE/g of dry sample.

\subsection{Statistical analysis}

Statistical analysis was performed by applying the JMP statistical software version 10 for Windows (SAS Institute INC., Cary, North California, USA). Data were subjected to analysis of 
variance (ANOVA) $(P<0.05)$. All performances were conducted at least in triplicate and all were averaged. The confidence limits used in this study were based on $95 \%(P<0.05)$.

\section{Results and Discussion}

\subsection{Effect of extraction temperature and time on TPC and AAs}

Statistical analysis expressed the effect of temperature and time on the phenolic extraction content (TPC) and the antioxidant activities (AAs). The results indicated that extraction time did not appear to significantly affect the TPC and the AA, whereas extraction temperature did have major effects in the TPC values and the AAs by the ABTS and FRAP assays. The AA of DPPH assays did not seem to be significantly affected by any individual or correlation effects of temperature and time.

Overall, as extraction temperature increased, the extraction efficiency, namely the phenolic extraction and antioxidant power by all assays, also increased (Figure 1). This experiment pattern was similar to that of other studies conducted by Spigno et al. (2007), Oancea et al. (2012), and Vergara-Salinas et al. (2012). Reportedly, an increased temperature during extraction could provide enough thermal energy to break down the interaction between solutes and the sample matrix. These internal forces could be the Van der Waals forces, hydrogen bonding, or the dipole attraction between the analyte molecules and the active sites in the matrix (Alupului et al., 2012). Furthermore, a high temperature could accelerate extraction yield by improving mass transfer rate of solutes out of the sample matrix towards the solution and reduce activation energy required for desorption process (Sparr Eskilsson \& Björklund, 2000; Vergara-Salinas et al., 2012).

The results indicated that TPC values (Figure 1a) of aqueous sesame extracts ranged between 5.57 and $6.77 \mathrm{mg}$ GAE/g of dry sesame cake which was highly comparable to that of other kinds of seed oil meal and by-products, and fruit peels in many studies. As the data comparison made sesame cake a potential source of natural antioxidants, the utilization of sesame meal could be practical in terms of phenolic extraction instead of a source of waste or fodder. Moreover, the higher TPC values of aqueous extracts of sesame cake than that of solvent extracts in many pub- lished studies (as discussed previously) also indicated that water could be used as an alternative solvent for other organic solvent in phenol extraction of sesame cake. This could potentially reduce the cost for extraction solvent as well as for evaporation or separation of solvent after the extract and make the sesame extracts more applicable and available especially in the food-based and health-related applications.

Antioxidant activities was measured from the sesame extract by using ABTS, DPPH and FRAP assays (Figure 4b, c and d, respectively). The average values of AA of each assay ranged from 16.57 to $19.20 \mathrm{mg}$ TE/g of dry sesame cake, from 3.69 to $3.95 \mathrm{mg} \mathrm{TE} / \mathrm{g}$ of dry sesame cake, and from 6.87 to $7.47 \mathrm{mg}$ TE/g of dry sesame cake for ABTS, DPPH and FRAP assays, respectively.

The ABTS $^{\bullet+}$-scavenging data indicates that antioxidant components of the sesame extract had ability to scavenge the free radicals via a mechanism of electron/hydrogen donation. The $\mathrm{ABTS}^{+}$scavenging activities of the sesame cake extracts was greater than that of $\mathrm{DPPH}^{+}$radical. Mohdaly et al. (2010) explained the possible reasons due to the stereoselectivity of radicals and the different solubility of extracts at different assays. Some compounds possessing $\mathrm{ABTS}^{+}$ scavenging activity in the extracts may not expose to show similar DPPH-scavenging activity. Floegel et al. (2011) noted that ABTS assays were more preferable to express the antioxidant contents than DPPH assays. ABTS assays could be employed in both hydrophilic and lipophilic solvent extraction while DPPH assays were only applicable in case of organic solvent which is most frequently under lipophilic conditions. In case of sesame cake extract, the oil-pressing process has effectively extracted. Hence, most of oil-soluble antioxidants was released out together with the sesame oil. Moreover, under the aqueous extraction, a vast majority of phenolics extracted were supposed to be more hydrophilic rather than lipophilic. Therefore, the aqueous conditions and the basic instinct of the two assays, ABTS assay was recorded for its better reflects the antioxidant contents than DPPH assay in case of sesame cake aqueous extracts.

$\mathrm{DPPH}^{\bullet}$ is considered as a stable of hydrophobic free radical which was employed to measure the free radical scavenging capacities of the extracts. DPPH radical scavenging activity of the extracts may be attributed to their hydrogen do- 


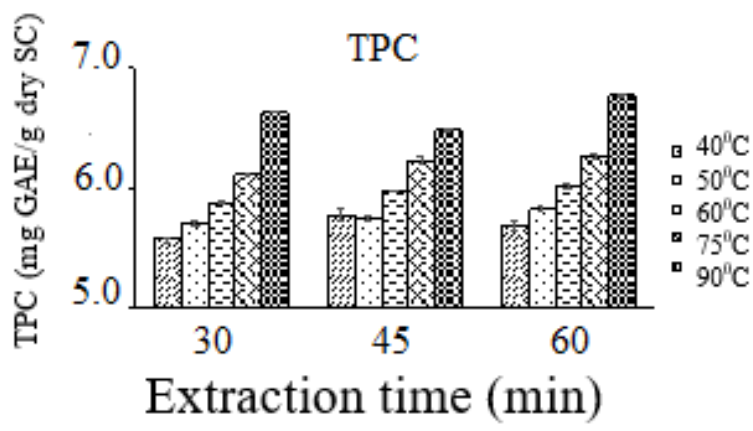

(a)

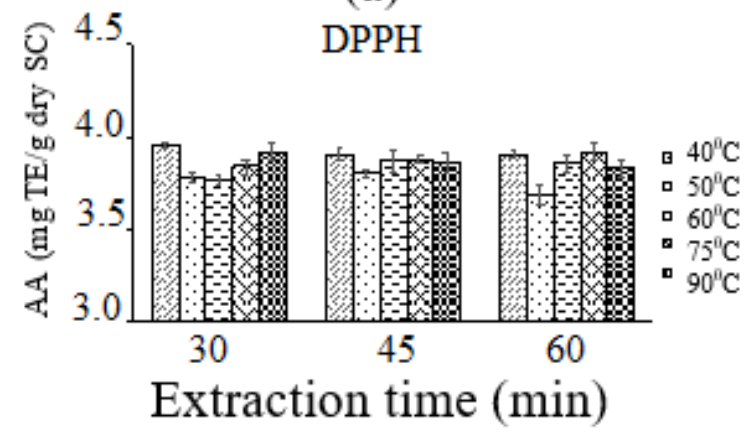

(c)

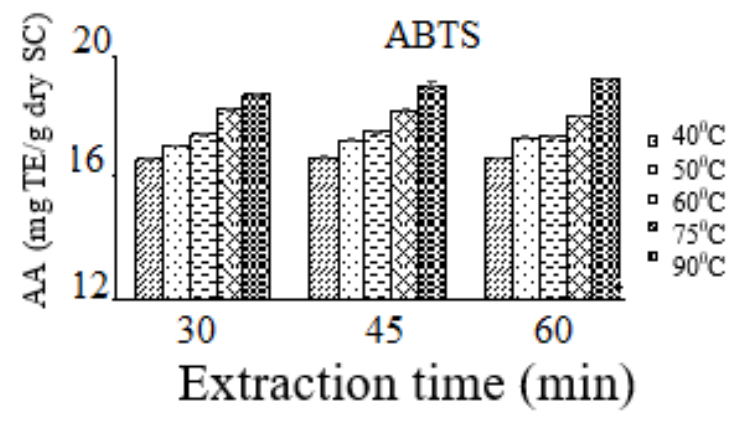

(b)

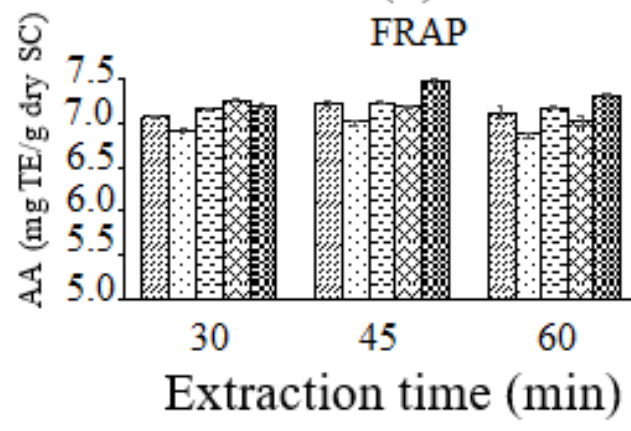

(d)

Figure 1. Effect of temperature and time on total phenolic content (TPC) and antioxidant activity (AA).

nation ability (Mohdaly et al., 2010; Reshma et al., 2013). As antioxidants in the extracts reacted with $\mathrm{DPPH}^{\bullet}$ radicals, the number of free radical decreased, resulting in the discoloration visually from dark-purple to yellow. The scavenging activity of extracts against $\mathrm{DPPH}^{\bullet}$ radical was concentration-dependent. Hence, higher results show greater antiradical capacity of the extracts.

In case of FRAP assay, under the presence of antioxidants acting as reductants, the ferrictripyridyltriazine ( $\mathrm{Fe}^{\mathrm{III}}$-TPTZ) complex is reduced to the ferrous $\left(\mathrm{Fe}^{\mathrm{II}}\right)$ form with an intense blue color (Benzie \& Strain, 1996). Results from antioxidant activities based on the FRAP assays showed that the sesame cake extracts did possess the ferric reducing capacity under the experiment conditions.

All responses obtained highest values at the experiments of $90^{\circ} \mathrm{C}$. Yet, only TPC values and AA values obtain from ABTS assays were significantly higher at $90^{\circ} \mathrm{C}$ compared to lower levels of temperature. The possible reasons that antioxidants possessing the ability to scavenge $\mathrm{DPPH}^{\bullet}$ radicals and their reducing power were not effectively involved to the increasing temperature and the net increase in their antioxidant values are not enough to be significant. Since time did not take into significant account to the overall effects during extraction, statistic results showed that the optimum extraction condition should be set at $90^{\circ} \mathrm{C}$ for $30 \mathrm{~min}$ for the optimal extraction efficiency.

Correlations of TPC and AAs of the sesame extracts:

Correlations between the TPC and the antioxidant activities (AA) by all assays were analyzed by using the Pearson's correlation analysis (Table 1). Only the correlation of TPC values and AA based on ABTS assay was significantly high $(\mathrm{r}=0.85, P<0.05)$. The positively high correlation informed that the concentration of phenolic extracts possibly plays major roles in the antioxidant activity (Candrawinata et al., 2014).

Mohdaly et al. (2010) suggested it was better to use $\mathrm{ABTS}^{+}$radical instead of $\mathrm{DPPH}^{\bullet}$ radical to determine the potential free radical scavenging activity of the sample in case that the issues of 


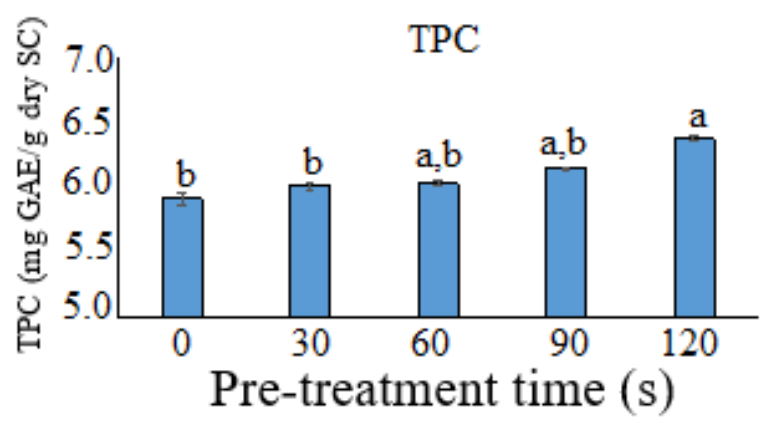

(a)

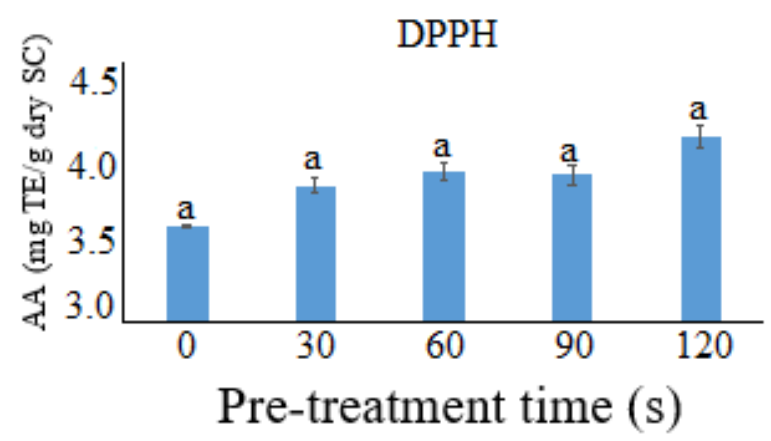

(c)

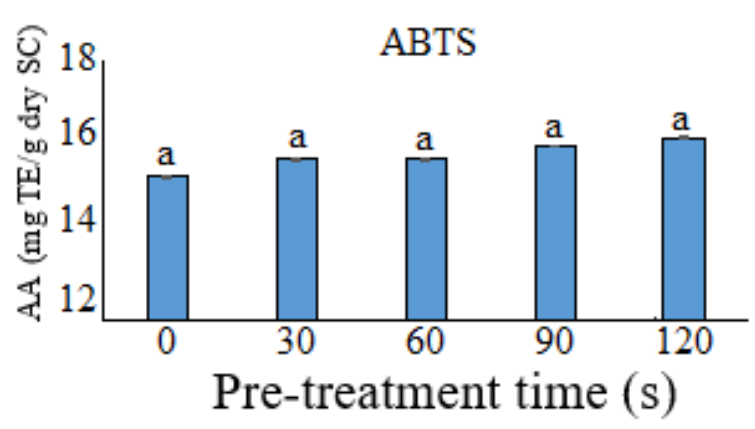

(b)

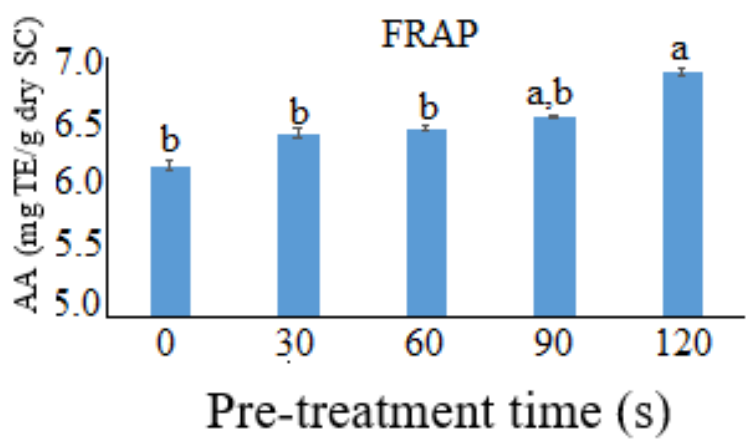

(d)

Figure 2. Effect of microwave pre-treatment time on TPC and AAs.

Values are means \pm standard errors for different extraction times and those not sharing a superscript are significantly different $(P<0.05)$.

Table 1. Pearson's correlation coefficients of TPC and AAs of the sesame cake extracts

\begin{tabular}{lccc}
\hline & TPC & ABTS & DPPH \\
\hline ABTS & $0.85^{*}$ & & \\
DPPH & 0.11 & -0.06 & \\
FRAP & 0.41 & 0.28 & 0.35 \\
\hline *: significant at $P<0.05$ & & &
\end{tabular}

sample solubility are a matter. The composition of antioxidants, different selectivity of radical and the solubility of extracts could influence the capacity of extract to react with other radicals in each assay (Mohdaly et al., 2010). As mentioned previously, the high hydrophobicity of $\mathrm{DPPH}^{\bullet}$ radicals led the assay less acceptable than the more flexible nature of ABTS under the aqueous extraction condition. Therefore, the significantly higher $r$ values between TPC x ABTS and TPC x DPPH and TPC x FRAP suggested that ABTS assays would be appropriate to determine quickly antioxidant activities from sesame cake aqueous extract. Nevertheless, none of the assay was recommended to be used alone to avoid interferences resulting in the underestimate or overestimate the overall antioxidant capacities of samples.

\subsection{Effects of pre-treatment by microwave on TPC and AA}

As the microwave power and frequency was preset at $700 \mathrm{~W}$ and $2450 \mathrm{~Hz}$ respectively, the pre-treatment time was the only independent parameters. Data showed it witnessed an increase in TPC and AA as the pre-treatment time by microwave irradiation increased (Figure 2). Even though all responses became progressively greater, statistical analysis indicated that only the TPC (Figure 2a) and AA based on FRAP (Figure 2d) assays was considerably improved as the samples were pre-treated by microwave irradiation for 120 s as compared with the non-pretreated. The $\mathrm{ABTS}^{\bullet+}$ (Figure 2b) and $\mathrm{DPPH}^{\bullet}$ 
(Figure 2c) scavenging capacity was not greatly elevated by the pre-treatment of microwave irradiation.

Microwave pre-treatment has been reported to have more significant support effects than ultrasound pretreatment in the extraction efficiency of sesame cake extracts. In an unpublished research, data showed ultrasound seems to have no significant role in improving the efficiency of extraction. Wataniyakul et al. (2012) did conclude microwave was possibly applied to treat samples before water extraction. The presence of water with rather high dielectric constant results in a phenomenon called "superheating" in which microwave energy were well absorbed by water in the matrix but poor dissipated. The cells got heating very fast and became ruptured to release more solutes which is partially antioxidants, leading to a higher extraction recoveries (Veggi et al., 2013).

Although results showed a significant increase in TPC values for pre-treated samples relative to the non-pretreated, that recoveries was not related to an increase in AA values of $\mathrm{DPPH}$ and ABTS assays, indicating that the free radical scavenging abilities of the extracts were either treated for long time enough that made the two treatment insignificant or the newly-extracted phenolics did not retain the scavenging ability. In case on FRAP assays, a significantly greater AA values at 120 s relative to other treatments showed the possible connection between microwave irradiation and reducing ability of antioxidant. Yet, there is little evidence to prove this case of phenomenon.

\section{Conclusion}

Polyphenol aqueous extraction from sesame cake proposed a promising replacement of organic solvents by water. It was the extraction temperature that had major influence on the TPC and antioxidant activities of the extracts while in the experiment range of present studies, extraction time was found to be insignificant. The optimum extraction condition recommended were $90^{\circ} \mathrm{C}$ for $30 \mathrm{~min}$ in this research. Moreover, microwave pre-treatment at 120 s could have significantly positive influence on the overall extraction yield, especially the total phenolics and antioxidants based on FRAP assays. All three assays showed the positive antioxidant activities of the extracts, ABTS assays, however, was suggested to be the most appropriate assays among the three assays. Moreover, the differences in antioxidant efficiencies are also implying that phenolic from the extracts are not the only contributor to the total antioxidant activities in the sesame cake. The obtained results, in the overall view, suggest that sesame aqueous extracts could be a source of antioxidants with more feasible applications in food as well as other industry. Further studies could focus on determining more appropriate antioxidant activities assays to obtain a more comprehensive understanding on antioxidant properties of the extracts.

\section{References}

Alupului, A., Călinescu, I., \& Lavric, V. (2012). Microwave Extraction of active principles from medicinal plants. UPB Scientific Bulletin, Series B: Chemistry and Materials Science 74, 129-142.

Arnao, M. B., Cano, A., \& Acosta, M. (2001). The hydrophilic and lipophilic contribution to total antioxidant activity. Food Chemistry 73(2), 239-244.

Azmir, J., Zaidul, I. S. M., Rahman, M. M., Sharif, K. M., Mohamed, A., Sahena, F., Jahurul, M. H. A, Ghafoor, K., Norulaini, N. A. N., \& Omar, A. K. M. (2013). Techniques for extraction of bioactive compounds from plant materials: A review. Journal of Food Engineering 117(4), 426-436.

Benzie, I. F. F., \& Strain, J. J. (1996). The Ferric Reducing Ability of Plasma (FRAP) as a Measure of "Antioxidant Power": The FRAP Assay. Analytical Biochemistry 239(1), 70-76.

Brand-Williams, W., Cuvelier, M. E., \& Berset, C. (1995). Use of a free radical method to evaluate antioxidant activity. LWT - Food Science and Technology 28(1), 25-30.

Çam, M., \& Aaby, K. (2010). Optimization of Extraction of Apple Pomace Phenolics with Water by Response Surface Methodology. Journal of Agricultural and Food Chemistry 58(16), 9103-9111.

Candrawinata, V. I., Golding, J. B., Roach, P. D., \& Stathopoulos, C. E. (2014). Optimisation of the phenolic content and antioxidant activity of apple pomace aqueous extracts. CyTA - Journal of Food 13(2), 293299.

Floegel, A., Kim, D. O., Chung, S. J., Koo, S. I., \& Chun, O. K. (2011). Comparison of ABTS/DPPH assays to measure antioxidant capacity in popular antioxidantrich US foods. Journal of Food Composition and Analysis 24(7), 1043-1048.

Guo, C., Yang, J., Wei, J., Li, Y., Xu, J., \& Jiang, Y. (2003). Antioxidant activities of peel, pulp and seed fractions of common fruits as determined by FRAP assay. Nutrition Research 23(12), 1719-1726. 
Joana Gil-Chávez, G., Villa, J. A., Fernando AyalaZavala, J., Basilio Heredia, J., Sepulveda, D., Yahia, E. M., \& González-Aguilar, G. A. (2013). Technologies for Extraction and Production of Bioactive Compounds to be Used as Nutraceuticals and Food Ingredients: An Overview. Comprehensive Reviews in Food Science and Food Safety 12(1), 5-23.

Mohdaly, A. A., Sarhan, M. A., Smetanska, I., \& Mahmoud, A. (2010). Antioxidant properties of various solvent extracts of potato peel, sugar beet pulp and sesame cake. Journal of the Science of Food and Agriculture 90(2), 218-226.

Oancea, S., Stoia, M., \& Coman, D. (2012). Effects of Extraction Conditions on Bioactive Anthocyanin Content of Vaccinium Corymbosum in the Perspective of Food Applications. Procedia Engineering 42, 489-495.

Pan, X., Niu, G., \& Liu, H. (2003). Microwave-assisted extraction of tea polyphenols and tea caffeine from green tea leaves. Chemical Engineering and Processing: Process Intensification 42(2), 129-133.

Pinelo, M., Rubilar, M., Jerez, M., Sineiro, J., \& Nunez, M. J. (2005). Effect of solvent, temperature, and solvent-to-solid ratio on the total phenolic content and antiradical activity of extracts from different components of grape pomace. Journal of Agricultural and Food Chemistry 53(6), 2111-2117.

Reshma, M. V., Namitha, L. K., Sundaresan, A., \& Ravi Kiran, C. (2013). Total Phenol Content, Antioxidant Activities and $\alpha$-Glucosidase Inhibition of Sesame Cake Extracts. Journal of Food Biochemistry 37(6), 723-731.

Saikia, S., Mahnot, N. K., \& Mahanta, C. L. (2015). Optimisation of phenolic extraction from Averrhoa carambola pomace by response surface methodology and its microencapsulation by spray and freeze drying. Food Chemistry 171(0), 144-152.

Sparr Eskilsson, C., \& Björklund, E. (2000). Analyticalscale microwave-assisted extraction. Journal of Chromatography A 902(1), 227-250.

Spigno, G., Tramelli, L., \& De Faveri, D. M. (2007). Effects of extraction time, temperature and solvent on concentration and antioxidant activity of grape marc phenolics. Journal of Food Engineering 81(1), 200-208.
Suja, K. P., Jayalekshmy, A., \& Arumughan, C. (2005). Antioxidant activity of sesame cake extract. Food Chemistry 91(2), 213-219.

Terpinc, P., Čeh, B., Ulrih, N. P., \& Abramovič, H. (2012). Studies of the correlation between antioxidant properties and the total phenolic content of different oil cake extracts. Industrial Crops and Products 39, 210-217.

Thaipong, K., Boonprakob, U., Crosby, K., CisnerosZevallos, L., \& Hawkins Byrne, D. (2006). Comparison of ABTS, DPPH, FRAP, and ORAC assays for estimating antioxidant activity from guava fruit extracts. Journal of Food Composition and Analysis 19(6-7), 669-675.

Veggi, P., Martinez, J., \& Meireles, M. A. (2013). Fundamentals of microwave extraction. In F. Chemat \& G. Cravotto (Eds.). Microwave-assisted extraction for bioactive compounds (15-52). New York, USA: Springer.

Vergara-Salinas, J. R., Perez-Jimenez, J., Torres, J. L., Agosin, E., \& Perez-Correa, J. R. (2012). Effects of temperature and time on polyphenolic content and antioxidant activity in the pressurized hot water extraction of deodorized thyme (Thymus vulgaris). Journal of Agriculture and Food Chemistry 60(44), 1092010929.

Wanasundara, U. N., Amarowicz, R., \& Shahidi, F. (1995). Partial characterization of natural antioxidants in canola meal. Food Research International 28(6), 525-530.

Wang, W., Ma, X., Xu, Y., Cao, Y., Jiang, Z., Ding, T., Xe, X., \& Liu, D. (2015). Ultrasound-assisted heating extraction of pectin from grapefruit peel: Optimization and comparison with the conventional method. Food Chemistry 178(0), 106-114.

Wataniyakul, P., Pavasant, P., Goto, M., \& Shotipruk, A. (2012). Microwave pretreatment of defatted rice bran for enhanced recovery of total phenolic compounds extracted by subcritical water. Bioresourse Technology $124,18-22$. 